\title{
¿Estamos involucrados en autenticar el conocimiento universal?
}

\section{Are we involved in authenticating universal knowledge?}

\begin{abstract}
Cinthia C. Cazal-Martínez ${ }^{1} *_{\mathbb{O}^{1}}^{1}$ Universidad Nacional de Asunción. Centro Multidisciplinario de Investigaciones Científicas y Tecnológicas. San Lorenzo, Paraguay. *Autor de correspondencia: ccazal@rec.una.py.
\end{abstract}

Recibido: 28 junio 2021

Aceptado: 9 julio 2021
Recibido en versión modificada: 6 julio 2021
Cazal-Martínez, C. C. (2021). ¿Estamos involucrados en autenticar el conocimiento universales? Investigaciones y Estudios - UNA, 12(1), 1-3.

https://doi.org/10.47133/IEUNA211e

En Mendoza \& Paravic (2006), se lee el siguiente párrafo mencionado por Krauskopf y Vera, 1995 si un país aspira construir un patrimonio intelectual, pilar de su propio progreso, no puede marginarse del desafio que implica dominar la tecnología que además de la creación y recreación del saber - debe validar pública y universalmente el nuevo conocimiento(...). En consecuencia, la publicación de Revistas Cientificas en países de América Latina donde existe una base científica mínima es imperativa. De lo contrario, el aporte de la comunidad de investigadores de competencia universal sería incompleto ya que no se incrementaría nuestra capacidad tecnológica para cubrir todo el proceso que involucra autentificar el conocimiento universal. Reflexionando al respecto, he encontrado que dichas afirmaciones se encuentran plasmadas en el progreso de las Ciencias en Paraguay en los últimos 10 años, a partir, de la vigencia de la Ley Nro. 4738/2012 - Que crea el Fondo Nacional de Inversión Pública y Desarrollo
(FONACIDE) y el Fondo para la Excelencia de la Educación y la Investigación (FEEI). Se han impulsado diversos programas y proyectos con el objetivo de elevar la calidad de la educación, la búsqueda de la excelencia educativa y la promoción de la investigación y el desarrollo (Secretaría Técnica de Planificación, 2012).

Puedo decir que siendo parte de este proceso existe un tímido, pero importante avance de la investigación en nuestro país. Gracias a estos fondos se ha generado conocimiento y consolidado diferentes grupos de trabajo ...cumpliendo con lo anterior "la creación y recreación del saber - debe validar pública y universalmente el nuevo conocimiento", cuyos aportes cumplen con altos estándares para ser publicados como artículos originales en revistas de impacto a nivel internacional, complementando "el aporte de la comunidad de investigadores de competencia universal" incrementando "nuestra capacidad tecnológica para cubrir todo el proceso que involucra autentificar el conocimiento universal".

La Revista Investigaciones y Estudios-UNA (RIEU), en su carácter de revista multidisciplinar dependiente de la Universidad Nacional de 
Asunción, se ha enfocado en hacer posible comunicar los resultados de investigaciones obtenidas dentro de las diferentes áreas de la ciencia. Para ello ha adoptado nuevas normativas para el cumplimiento de criterios que eleven la visibilidad de la comunicación cientifica nacional y regional.

Como una Revista Científica en un país de América Latina, dependiente de una Universidad Pública, donde la generación de nuevos conocimientos es constante y de alta calidad, pero con los recursos financieros destinados a la investigación limitados busca sumar y ser partícipe de esta necesidad "la publicación de Revistas Científicas en países de América Latina donde existe una base cientifica mínima es imperativa". Donde la RIEU adoptó como un desafío primordial, que es ser parte de la comunicación científica en lengua española, sin costos de acceso y publicación, disponible en línea para la comunidad científica nacional donde se pueda depositar esa base científica mínima necesaria para la región. A partir de ello consolidarse como una revista multidisciplinar y una alternativa más para que investigadores de América Latina publiquen sus aportes. Con énfasis al fortalecimiento de la formación de nuevos investigadores, que se hacen camino siendo parte de Programas de Iniciación Científica y de Posgrados, garantizando que sus postulaciones recibirán evaluaciones con críticas constructivas que colaboren al desarrollo de sus habilidades en la redacción científica.

Seguimos haciendo un repaso de la situación actual y se siguen proponiendo cambios dentro de la política editorial de la Revista Investigaciones y Estudios de la UNA, a fin de persistir y cumplir los ideales de indexación de la revista.
Durante este ultimo año 2020, un importante número de artículos originales de calidad que abordan temáticas de gran interés para los profesionales e investigadores de los diferentes ámbitos científicos han sido recepcionados. Y parte de dichos aportes se presenta en este primer número del Volumen 12 del año 2021, les presentamos trabajos enfocados en Ciencias Ambientales y Agrarias como, la problemática de la calidad de agua en cuencas hídricas que circulan en zonas urbanas y una revisión bibliográfica de calidad científica existente para el monitoreo de enfermedades fúngicas en el cultivo de Soja en Paraguay, respectivamente. Dentro del área de Ciencias Médicas se incluyen dos aportes de estudios descriptivos sobre niños $\mathrm{y}$ adolescentes con trastornos del espectro autista y sobre lactancia materna exclusiva.

Así mismo, para los números previstos este año 2021, se ha decidido incluir dentro de los artículos publicados una sección de temas de actualidad con un eje temático denominado "Gestión y reducción de riesgos de desastres (GRRD)".

La GRRD, plantea las estrategias de abordaje de la gestión del riesgo de desastres a nivel nacional, regional/departamental, local o institucional. Están incluidas dentro de estas estrategias las enfocadas en: Cambios que genera la variabilidad climática y sus impactos en el sistema económico y social. Gestión y manejo sustentable de los recursos naturales como medida de prevención de desastres con el uso de tecnologías de control de contaminación a la situación de espacios urbanos e industriales. Estudio de amenazas naturales (inundaciones pluviales, fluviales y sequias), estudio de cuencas, sistemas de alerta temprana, incendios forestales. Saneamiento: Tratamiento de efluentes, 
Gestión de residuos. Psicología de la emergencia: resiliencia en las comunidades, experiencias de intervención en crisis. Dentro de la educación superior: formación, investigación y extensión, entre otros.

Por último dar mi reconociendo y agradecimiento a todas las personas que han enviado sus manuscritos a la revista y a los que han colaborado en las revisiones con una inmejorable predisposición y adaptándose a los plazos, como también a los miembros del comité editorial que han contribuido a nuestra tarea común de mejorar la comunicación científica.

\section{REFERENCIAS BIBLIOGRÁFICAS}

Mendoza, S., \& Paravic, T. (2006). Origen, Clasificación y desafíos de las Revistas Científicas. Investigacion y Postgrado,21(1), 49-

75.http://www.redalyc.org/articulo.oa?id $=64750104$ Secretaría Técnica de Planificación. (2012). Ley Nro. 4738. Que crea el Fondo Nacional de Inversión Pública y desarrollo (FONACIDE) y el Fondo para la excelencia de la Educación $y$ y la Investigación. https://nube.stp.gov.py/index.php/s/rQZG MW6GBwFVhhJ\#pdfviewer 